\title{
Artroplastia total
}

\section{trapézio-metacarpiana. Resultado a curto prazo de 2 implantes diferentes}

\author{
R. Claro ${ }^{(1)}$, A. Vilaça $^{(1)}$, P. Negrão ${ }^{(2)}$, M. Trigueiros ${ }^{(2)}$, C. Silva $^{(3)}$ \\ Serviço de Ortopedia. Hospital Geral de Santo antónio. Porto. Portugal

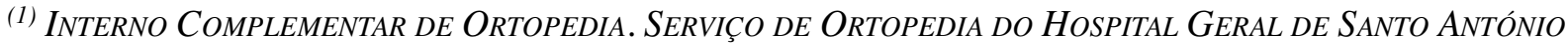 \\ (2) AsSIITENTE HospitaLAR DE ORTOPEDIA. SERVIÇO DE ORTOPEDIA Do HosPITAL GERAL DE SANTO ANTÓNIO \\ (3) Assistente Hospitalar Graduado de Ortopedia. Serviço de Ortopedia do Hospital Geral de \\ SANTO ANTÓNIO
}

Correspondência:

Dr. Rui Claro

Serviço de Ortopedia. Hospital Geral de Santo António

Largo Professor Abel Salazar

4099-001 Porto

Teléf. 222077500 - Fax. 222087632

e-mail: ruiclaromed@yahoo.com

A rizartrose é uma patologia muito comum que afecta frequentemente mulheres de meia-idade e por norma bilateralmente.

Muitas técnicas cirúrgicas têm vindo a ser descritas para o tratamento da rizartrose em estádios avançados, como é o caso da artrodese, da trapezectomia com ou sem interposição de tendão e/ou reconstrução ligamentar, dos implantes de silicone e das próteses totais trapézio-metacarpianas.

Os autores pretendem com este estudo comparar 2 tipos diferentes de implantes analisando retrospectivamente a utilização destes no tratamento de 15 doentes com rizartrose bilateral.

Na série estudada, a prótese trapézio-metacarpiana provou ser eficaz no alívio sintomático e no aumento da mobilidade e da força do polegar. $\mathrm{O}$ implante do tipo «ball-and-socket» obteve melhores resultados funcionais e um menor tempo de recuperação, em relação à prótese «anatómica».

Palavras chave: rizartrose, articulação trapéziometacarpiana, prótese trapézio-metacarpiana.
Osteoarthritis of the thumb carpometacarpal joint is a common disorder in postmenopausal woman and is usually bilateral.

Diverse techniques have been described for the surgical treatment in the advanced stages of thumb osteoarthritis, such as arthrodesis, complete excision of the trapezium with or without tendon interposition and ligament reconstruction, silicone implants and total trapeziometacarpal joint arthroplasty.

The authors present a study of two different prosthesis used for the treatment in 15 patients with bilateral thumb carpometacarpal joint osteoarthritis. In our group of patients total trapeziometacarpal joint arthroplasty has proven to be effective for pain relief, improved motion and strength. The «balland-socket» implants had better functional results and a short period of rehabilitation, compared to «anatomical» implants.

Key words: thumb osteoarthritis, trapeziometacarpal joint, prosthesis. 

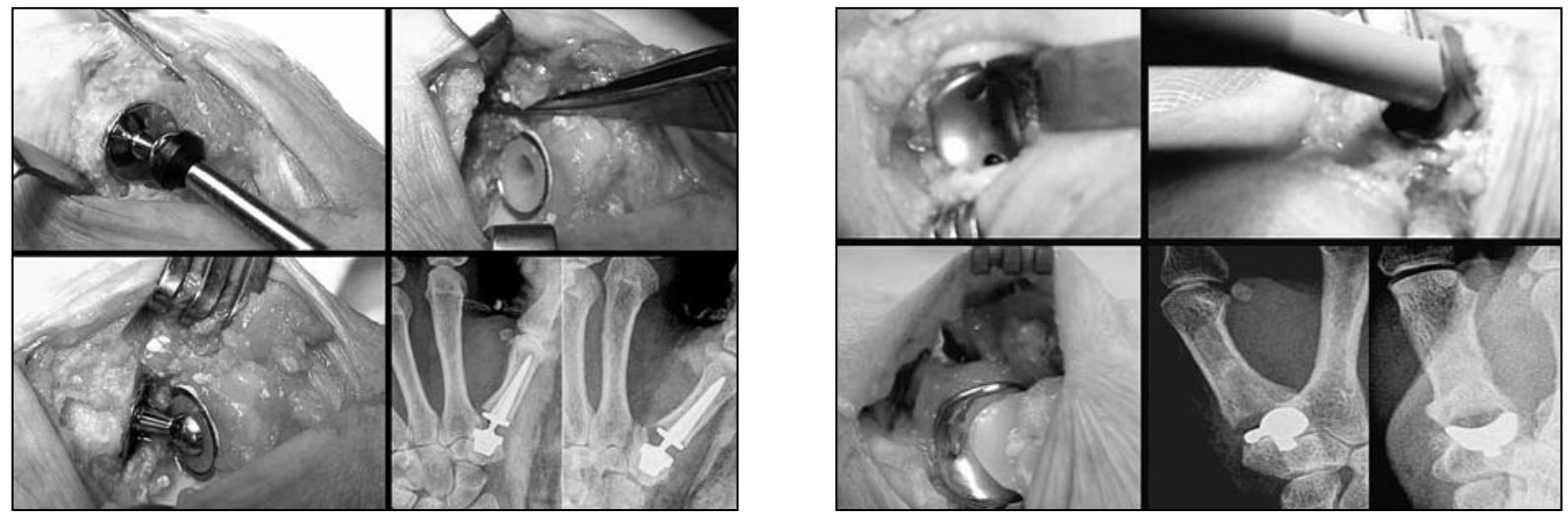

Figura 1. Pormenor da técnica cirúrgica das próteses. A: Roseland ${ }^{\circledR}$ e B: $S R^{T M} T M C$.

\section{INTRODUÇÃO}

A artrose da articulação trapézio-metacarpiana (ou rizartrose) é uma patologia muito comum que afecta frequentemente mulheres de meia-idade e por norma bilateralmente ${ }^{1,2}$.

Muitas técnicas cirúrgicas têm vindo a ser descritas para o tratamento da rizartrose em estádios avançados, como é o caso da artrodese, da trapezectomia com ou sem interposição de tendão e/ou reconstrução ligamentar, dos implantes de silicone e das próteses totais trapézio-metacarpianas ${ }^{3-6}$.

Uma das vantagens da prótese em relação aos outros procedimentos é a obtenção de uma articulação indolor, estável e com boa mobilidade num polegar com normal comprimento. A prótese de de la Caffinière, desenvolvida no início da década de $1970^{7}$, foi um dos primeiros implantes a ser utilizado universalmente. Actualmente está disponível uma grande variedade de implantes, quanto ao seu design, material de fabrico e métodos de fixação.

A trapezectomia com tendinoplastia de interposição e ligamentoplastia tem sido a técnica de referência da Unidade de Cirurgia de Mão do Hospital Geral Santo António no tratamento cirúrgico da rizartrose ${ }^{3-4}$. Nos últimos anos, tem vindo a ser progressivamente utilizada, por esta Unidade, a prótese total trapézio-metacarpiana em doentes mais idosos, com actividades de vida diárias menos exigentes e que necessitam de um polegar indolor, estável, com boa mobilidade e com um tempo de recuperação o mais curto possível.
Os autores pretendem com este estudo comparar 2 tipos diferentes de implantes analisando retrospectivamente a utilização destes no tratamento de doentes com rizartrose bilateral.

\section{MATERIAL E MÉTODO}

Foram analisadas 15 próteses totais trapéziometacarpianas em 15 doentes com rizartrose bilateral (estádios III e IV de Eaton-Littler), realizadas entre Janeiro de 2005 e Fevereiro de 2007.

As indicações para cirurgia, após falência do tratamento conservador, foram: a dor refractária, a perda de força de preensão e de pinça, e a diminuição da mobilidade do polegar condicionando limitação das actividades diárias.

O tempo médio de seguimento foi de 12 meses (variação: 10 a 24 meses). Em 8 doentes foram utilizados implantes cimentados «anatómicos» $\mathrm{SR}^{\text {TM }}$ TMC - Avanta Orthopaedics; e em 7 doentes a prótese do tipo «ball-and-socket» não-cimentada constritiva Roseland ${ }^{\circledR}$ - DePuy. Dez implantes foram realizados no membro dominante e 5 no membro não dominante (Figura 1). Todos os doentes são do sexo feminino, com uma idade média, na altura da cirurgia, de 68,5 anos (variação: 62 a 80). Dez doentes são empregadas domésticas e as restantes 5 eram reformadas aquando da cirurgia.

$\mathrm{O}$ resultado do tratamento foi avaliado segundo critérios clínicos e radiológicos. A avaliação clínica foi baseada em 4 itens: dor, função, mobilidade e força. A avaliação radiológica foi realizada no pré e no pós-operatório, com 


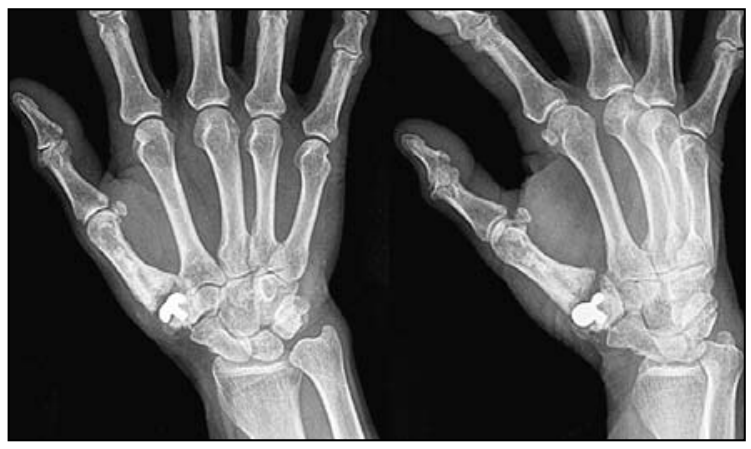

Figura 2. Rx de face e perfil da articulação trapézio-metacarpiana. Prótese «anatómica»-SR ${ }^{T M} T M C$.

incidências de face e perfil da articulação trapézio-metacarpiana (TMC).

A dor foi medida utilizando uma escala visual de dor, variando entre 0 e 10 pontos, correspondendo a ausência de dor e dor máxima respectivamente.

A avaliação funcional baseou-se na possibilidade de executar actividades da vida diária como: apanhar moedas, abrir uma tampa de garrafa, rodar uma chave na fechadura, abotoar/ desabotoar uma peça de roupa e escrever.

A mobilidade da articulação TMC foi medida em 3 direcções: abdução radial, extensão e oposição do polegar ${ }^{8}$.

Em todos os doentes foi medido a força de preensão e de pinça utilizando o mesmo dinamómetro (Jamar ${ }^{\circledR}$ Hand Dynamometer - 5030J1).

De acordo com os critérios de Eaton-Littler ${ }^{5}$ 12 doentes foram classificados como grau III e 3 como grau IV. O estudo imagiológico no pósoperatório permitiu avaliar a colocação dos implantes e a presença/ausência de linhas de radiolucência (Figura 2 e 3).

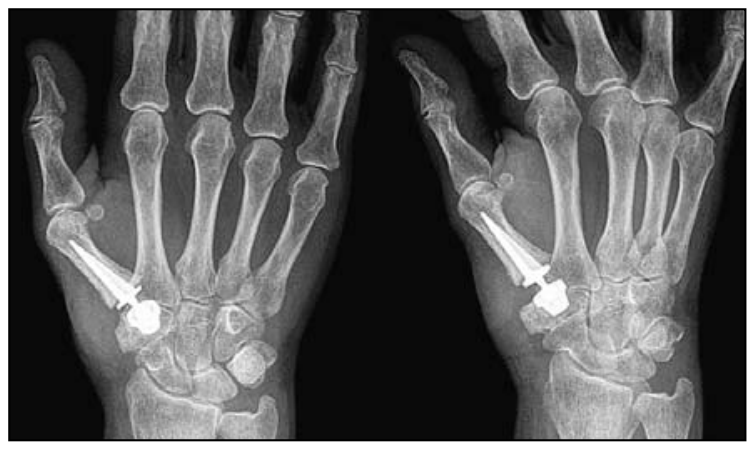

Figura 3. Rx de face e perfil da articulação trapézio-metacarpiana. Prótese «ball-and-socket»-Roseland ${ }^{\circledR}$.

\section{RESULTADOS}

Todos os doentes foram submetidos a uma avaliação clínica e radiológica através de uma consulta de revisão.

Em relação à mobilidade do polegar operado, todas as doentes conseguiram realizar o movimento de oposição até à base do $5^{\circ}$ dedo; e verificou-se um aumento do arco de mobilidade (cerca de $15^{\circ}$ na abdução radial e $10^{\circ}$ na extensão) (Quadro I).

A força de preensão média da mão operada foi de $24,3 \mathrm{Kg}$ em doentes submetidos a próteses do tipo «ball-and-socket» e de $20,8 \mathrm{Kg}$ em doentes submetidos a próteses do tipo «anatómico»; sendo em média, na mão não-operada 19,4 $\mathrm{Kg}$ e 19,2 Kg respectivamente (Quadro II).

A força de pinça média da mão operada foi de 5,2 Kg em doentes submetidos a próteses do tipo «ball-and-socket» e de 4,7 Kg em doentes submetidos a próteses do tipo «anatómico»; sendo em média, na mão não-operada $4,2 \mathrm{Kg}$ e 4,0 Kg respectivamente (Quadro III).

\section{Quadro I - MOBILIDADE DO POLEGAR}

\begin{tabular}{|c|c|c|c|c|c|}
\hline \multirow{2}{*}{} & \multicolumn{4}{|c|}{ Pós-op } \\
\cline { 3 - 6 } & & \multicolumn{2}{|c|}{ SR TMC } & \multicolumn{2}{c|}{ ROSELAND® } \\
\cline { 3 - 6 } & Mão operada & Mão n-operada & Mão operada & Mão n-operada \\
\hline \multirow{2}{*}{ Abdução } & Média & $40^{\circ}$ & $25^{\circ}$ & $45^{\circ}$ & $30^{\circ}$ \\
\cline { 2 - 6 } & Variação & $35-45$ & $20-40$ & $35-50$ & $20-40$ \\
\hline \multirow{2}{*}{ Extensão } & Média & $15^{\circ}$ & $5^{\circ}$ & $15^{\circ}$ & $5^{\circ}$ \\
\cline { 2 - 6 } & Variação & $5-10$ & $3-10$ & $5-10$ & $3-10$ \\
\hline
\end{tabular}


Todos apresentaram diminuição significativa da dor na última consulta de seguimento (Quadro IV).

De um modo global os doentes referiram serem capazes de executar as actividades da vida diária com menor dificuldade (Quadro V).

No que se refere ao índice de satisfação, 13 doentes estão satisfeitos ou muito satisfeitos com o resultado final. A diminuição da dor e a restituição da mobilidade são os principais motivos de satisfação. Setenta e cinco porcento desejam ser operados à mão contra-lateral $\mathrm{e}$ $85 \%$ recomendariam a cirurgia.
Observou-se uma fractura do trapézio durante a colocação do componente trapeziano da prótese do tipo «ball-and-socket». Optou-se, neste caso, por manutenção do implante e imobilização do polegar durante 6 semanas. Verificou-se consolidação do trapézio e um bom resultado funcional.

Não existiu nenhum caso de infecção ou de luxação da prótese.

Predomínio de um menor tempo de recuperação/reabilitação nos doentes submetidos a próteses do tipo «ball-and-socket», em média cerca de 3 meses até ao retorno às actividades

\section{Quadro II - FoRÇA DE PREENSÃO}

\begin{tabular}{|c|c|c|c|c|}
\hline \multirow{2}{*}{} & \multicolumn{4}{|c|}{ SRós-op } \\
\cline { 2 - 5 } & \multicolumn{2}{|c|}{ SR $^{\text {TM }}$ TMC } & \multicolumn{2}{c|}{ ROSELAND ${ }^{*}$} \\
\cline { 2 - 5 } & Mão operada & Mão n-operada & Mão operada & Mão n-operada \\
\hline Média (Kgf) & 20,8 & 19,2 & 24,3 & 19,4 \\
\hline Variação & $16-28$ & $12-26$ & $18-30$ & $14-28$ \\
\hline
\end{tabular}

\section{Quadro III - FORÇA DE PINÇA}

\begin{tabular}{|c|c|c|c|c|}
\hline \multirow{2}{*}{} & \multicolumn{3}{|c|}{ SR ${ }^{\text {TM }}$ TMC } & \multicolumn{2}{c|}{ ROSELAND (} \\
\cline { 2 - 5 } & Mão operada & Mão n-operada & Mão operada & Mão n-operada \\
\cline { 2 - 5 } & 4,7 & 4,0 & 5,2 & 4,2 \\
\hline Média (Kgf) & $13-7$ & $1,5-5,5$ & $14,5-7$ & $2,5-6$ \\
\hline Variação & \multicolumn{2}{|c|}{} &
\end{tabular}

\section{Quadro IV - AVALIAÇÃO DA DOR DO POLEGAR}

\begin{tabular}{|c|c|c|c|c|}
\hline \multirow{2}{*}{} & \multicolumn{2}{|c|}{ Pré-op } & \multicolumn{2}{c|}{ Pós-op } \\
\cline { 2 - 5 } & SR $^{\text {TM }}$ TMC & ROSELAND® & SR $^{\text {TM }}$ TMC & ROSELAND $\mathbb{~}$ \\
\hline Sem Dor & - & - & 4 & 6 \\
\hline Dor à mobilização & 8 & 7 & 3 & 1 \\
\hline Dor em repouso & 5 & 4 & 1 & - \\
\hline Dor nocturna & 4 & 3 & - & - \\
\hline Escala Subjectiva de Dor $(0-10$ & 7,5 & 8 & 3,5 & 2,6 \\
\hline
\end{tabular}


Quadro V - ACTIVIDADES DA VIDA DIÁRIA

\begin{tabular}{|c|c|c|c|c|}
\hline \multirow{2}{*}{} & \multicolumn{2}{|c|}{ Sem dificuldade } & \multicolumn{2}{c|}{ Com dificuldade } \\
\cline { 2 - 5 } & SR $^{\text {TM }}$ TMC & ROSELAND $\mathbb{1}$ & SR $^{\text {TM }}$ TMC & ROSELAND® \\
\hline Apanhar moedas & 6 & 6 & 3 & 1 \\
\hline Abrir tampa garra & 8 & 6 & 3 & 1 \\
\hline Rodar Chave & 3 & 3 & 4 & 2 \\
\hline Escrever & 5 & 5 & 2 & - \\
\hline Uso de faca & 5 & 4 & 2 & 1 \\
\hline
\end{tabular}

de vida diária, em relação aos doentes submetidos a próteses do tipo «anatómico», em média cerca de 7 meses até ao retorno às actividades de vida diária.

Dois doentes foram submetidos a cirurgia de revisão, o primeiro por descelagem traumática dos implantes (queda ao mesmo nível com traumatismo directo da mão no $5^{\circ}$ mês de pós-operatório) (Figura 4); e o segundo por má colocação do componente trapeziano (dor incapacitante durante todo o pós-operatório) (Figura 5). Os dois implantes em causa foram do tipo «anatómico». Em ambos os casos foi realizada cirurgia de revisão com extracção dos implantes, trapezectomia e ligamentoplastia de suspensão, com resultado funcional final satisfatório.

O estudo radiográfico não revelou sinais de descelagem nos restantes implantes.

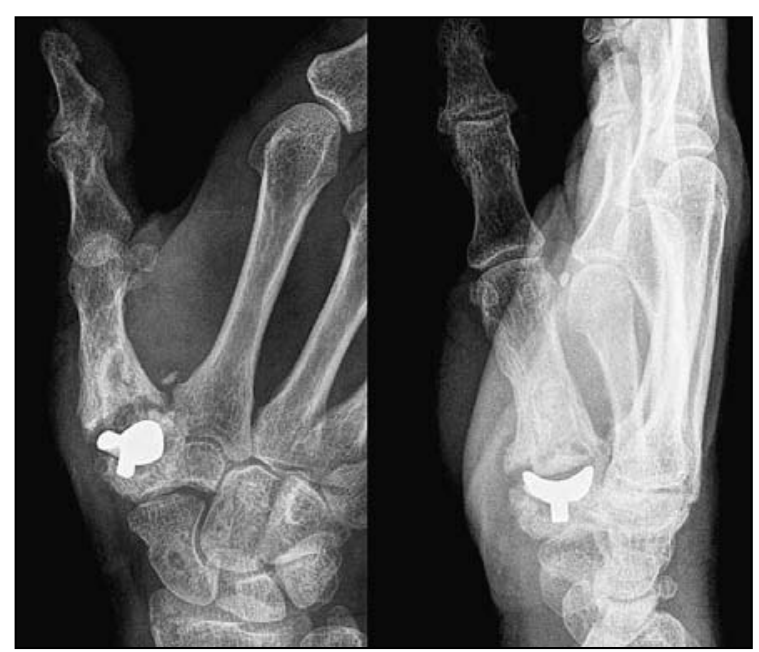

Figura 4. Rx de face e perfil da articulação trapézio-metacarpiana. Descelagem traumática do componente trapeziano da prótese $S R^{T M} T M C$.

\section{DISCUSSÃO}

O principal objectivo no tratamento cirúrgico da rizartrose é a eliminação da dor, num polegar estável, procurando o restabelecimento da função $^{6-11}$. Tal é obtido com a restituição da estabilidade e mobilidade do polegar, e secundariamente com a recuperação da força.

Apesar de se conseguir um polegar indolor e estável com a artrodese da articulação TMC, esta opção cirúrgica acarreta um longo período de imobilização e uma diminuição marcada da função da mão ${ }^{12}$.

A trapezectomia isolada, embora preserve a mobilidade, condiciona uma diminuição do comprimento da coluna do polegar e por norma instabilidade. Quando associada a interposição de tendão e/ou reconstrução ligamentar,

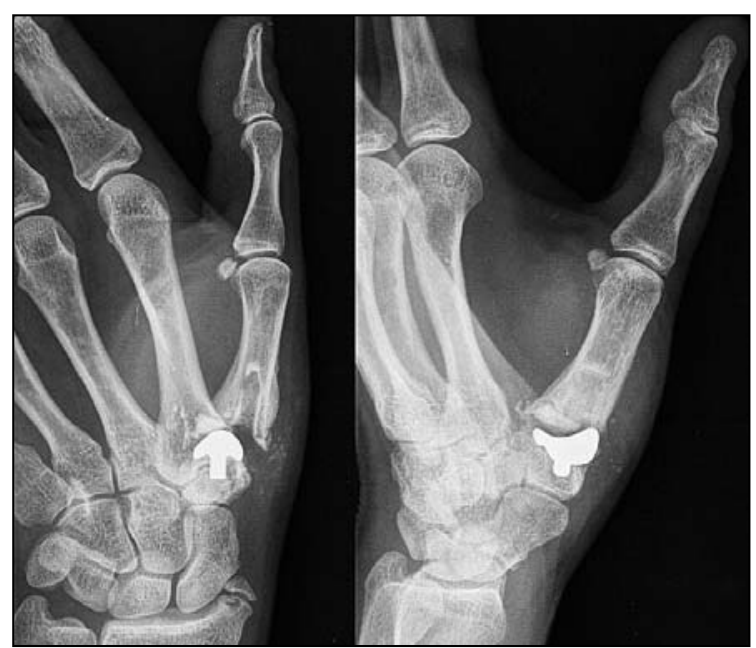

Figura 5. Rx de face e perfil da articulação trapézio-metacarpiana. Má colocação do componente trapeziano da prótese $S R^{T M} T M C$.

Revista Iberoamericana de Cirugía de la Mano 
estes problemas são potencialmente diminuídos. Porém, esta técnica necessita de um longo período de reabilitação ${ }^{3-4}$.

Existem inúmeros implantes para a articulação TMC, cimentados e não cimentados e com diferentes designs. Baseiam-se essencialmente em duas diferentes concepções: a artroplastia de interposição e a artroplastia total da articulação TMC. No que respeita aos implantes utilizados na artroplastia total, estes podem ser do tipo «anatómicos» que mimetizam as superfícies articulares; e implantes do tipo «ball-andsocket» na sua grande maioria constritivos ${ }^{9}$. Os defensores dos implantes «anatómicos», como é o caso da prótese $\mathrm{SR}^{\mathrm{TM}} \mathrm{TMC}$, baseiam-se na normal cinemática da articulação e na menor ressecção óssea. Os defensores dos implantes «ball-and-socket», como é o caso da prótese Roseland ${ }^{\circledR}$, procuram a estabilidade e a mobilidade da neo-articulação. No entanto, e apesar da crescente utilização deste tipo de implantes, a literatura aponta para taxas de falência apreciáveis, entre os $10 \%{ }^{10}$ e os $46 \%{ }^{11}$.

De um modo global, apesar do número reduzido de casos e do curto seguimento, a pró- tese «ball-and-socket», embora tecnicamente mais exigente, obteve melhores resultados funcionais, com um mais curto tempo de recuperação. Provavelmente por ser uma prótese intrinsecamente estável, obtendo um polegar estável de imediato, com um tempo de recuperação muito curto. O que não se verifica na prótese «anatómica», necessitando de um maior tempo de recuperação/reabilitação e com um menor resultado funcional.

\section{CONCLUSÃO}

Os autores consideram a artroplastia total da articulação TMC uma opção válida em doentes idosos, com rizartrose em estádios avançados e com pouca actividade física.

$\mathrm{Na}$ série estudada, a prótese TMC provou ser eficaz no alívio sintomático e no aumento da mobilidade e da força do polegar. O implante do tipo «ball-and-socket» obteve melhores resultados funcionais e um menor tempo de recuperação, em relação à prótese «anatómica», a curto prazo.

\section{BIBLIOGRAFÍA}

1. Pellegrini VD Jr. Osteoarthritis at the base of the thumb. Orthop Clin North Am, 1992; 23: 83-102.

2. Barron OA, Glickel SZ, Eaton RG. Basal joint arthritis of the thumb. J Am Acad Orthop Surg, 2000; 8: 314-23.

3. Silva C, Botelheiro J, Lemos R, et al. Rizartrose - Estudo comparativo da trapezectomia associada ou não a ligamentoplastia. Rev Port Ortop Traum, 1998; 6: 21-6.

4. Silva C, Lemos R, Gonçalves I. Tratamento cirúrgico da rizartrose: experiência e resultados da trapezectomia e ligamentoplastia. Rev Port Reumatol, 1997; 8: 2041-8.
5. Eaton RG, Lane LB, Littler JW, et al. Ligament reconstruction for the painful thumb carpometacarpal joint: a long-term assessment. J Hand Surg Am, 1984; 9: 692-9.

6. de la Caffinière JY. Prothèse totale trapézo-métacarpienne. Rev Chir Orthop, 1973; 59: 299-308.

7. Kapandji A. Cotation Clinique de l'opposition et de la contreopposition du pouce. An Chir Main, 1986; 5: 67-73.

8. van Capelle HGJ, Elzenga $P$, von Horn JR. Long-term results and loosening analysis of the De la Caffinière replacements of the trapeziometacarpal joint. J Hand Surg Am, 1999; 24: 476-82.
9. Pérez-Úbeda MJ, García-López A, Martínez FM, et al. Results of the cemented SR trapeziometacarpal prosthesis in the treatment of thumb carpometacarpal osteoarthritis. J Hand Surg Am, 2003; 28: 917-25.

10. Nicholas RM, Calderwood JW. De la Caffinière arthroplasty for basal thumb joint osteoarthritis. J Bone Joint Surg Br, 1992; 74: 309-12.

11. Wachtl SW, Sennwald GR. Noncemented replacement of the trapeziometacarpal joint. J Bone Joint Surg Br, 1996; 78: 787-92.

12. Müller GM. Arthrodesis of the trapezio-metacarpal joint for osteoarthritis. J Bone Joint Surg Br, 1949; 31: 540-2. 